\title{
On The Uniformisation of Algebraic Curves of Genus 3.
}

\author{
By M. Mursi.
}

(Received 3rd January 1930. Read 7th February 1930.)

\section{\$1. Introduction.}

An algebraic equation

$$
f(s, z)=0
$$

determines, in general, $s$ as a many valued function of $z$. If $s$ and $z$ can be expressed as one valued functions of a third variable $t$, then $t$ is called the uniformising variable. As Poincaré showed, $s$ and $z$ are automorphic functions of $t$.

For the hyperelliptic case where equation (1) is of the form

$$
s^{2}=\left(z-e_{1}\right)\left(z-e_{2}\right) \ldots\left(z-e_{2 n+2}\right),
$$

where $n>1$, the uniformising variable is the quotient of two solutions of ${ }^{1}$

$$
\begin{aligned}
& \frac{d^{2} y}{d z^{2}}+\frac{3}{16}\left\{\sum_{r=1}^{2 n+2} \frac{1}{\left(z-e_{r}\right)^{2}}\right. \\
& \left.+\frac{-(2 n+2) z^{2 n}+2 n p_{1} z^{2 n-1}+c_{1} z^{2 n-2}+\ldots+c_{2 n-1}}{\left(z-e_{1}\right)\left(z-e_{2}\right) \ldots\left(z-e_{2 n+2}\right)}\right\} y=0
\end{aligned}
$$

where $p_{2}=\sum_{r=1}^{2 n+2} e_{r}$

and the $c_{r}$ 's are constants whose values have never yet been found but which are theoretically to be determined by the condition that the group of equation (2) is to be fuchsian.

Dr J. M. Whittaker ${ }^{2}$ has suggested that the true equation (satisfying the condition that its group is fuchsian) is

where

$$
\frac{d^{2} y}{d z^{2}}+\frac{3}{16}\left[\left(\frac{\phi^{\prime}(z)}{\phi(z)}\right)^{2}-\frac{2 n+2}{2 n+1} \frac{\phi^{\prime \prime}(z)}{\phi(z)}\right] y=0
$$

$$
\phi(z)=\left(z-e_{1}\right)\left(z-e_{2}\right) \ldots\left(z-e_{2 n+2}\right) .
$$

The group of this equation for the case of the functions defined by $s^{2}=z^{5}+1$, which are of genus two, was actually calculated by Prof. Whittaker ${ }^{3}$ and proved to be a fuchsian group.

\footnotetext{
${ }^{1}$ Cf. E. T. Whittaker, Phil. Trans., Royal Soc. (A) 192 (1899), 1.

2 Journal London Math. Soc. 5 (1930).

3 Journal London Math. Soc. 4 (1929), 274.
} 
The object of the present note is to show that equation (3) also gives a fuchsian group for a case in which $n=3$ by calculating the generating transformations of the group. The algebraic form taken is

$$
s^{2}=1+z^{7}
$$

which is of genus 3 . The process in its essentials runs parallel with the case worked out by Prof. Whittaker for the form $s^{2}=1+z^{5}$.

§2. The hypergeometric form of equation (3).

Equation (3) has the same form for the curve $s^{2}=1+z^{2 n+1} \equiv \phi(z)$ as for the curve $s^{2}=1+z^{2 n+2}$, which has the same number of branch points.

Changing the dependent variable by the relation

$$
y=u\{\phi(z)\}^{\frac{1}{4}}
$$

(since we are concerned only with the ratio of two solutions we can multiply $y$ by any convenient function of $z$ ), equation (3) takes the form

$$
\phi(z) \frac{d^{2} u}{d z^{2}}+\frac{1}{2} \phi^{\prime}(z) \frac{d u}{d z}+\frac{1}{4}\left(1-\frac{3}{4} \frac{2 n+2}{2 n+1}\right) \phi^{\prime \prime}(z) u=0 .
$$

Now let us change the independent variable from $z$ to $s$ where

we then obtain

$$
s^{2}=1+z^{2 n+1} ;
$$

$$
(2 n+1)\left(s^{2}-1\right) \frac{d^{2} u}{d s^{2}}+4 n s \frac{d u}{d s}+2 n\left(1-\frac{3}{4} \frac{2 n+2}{2 n+1}\right) u=0 .
$$

If again we change from $s$ to $x$, where $s=2 x-1$, we get

$$
(2 n+1)(x-1) x \frac{d^{2} u}{d x^{2}}+2 n s \frac{d u}{d s}+2 n\left(1-\frac{3}{4} \frac{2 n+2}{2 n+1}\right) u=0,
$$

or, finally,

$$
x(x-1) \frac{d^{2} u}{d x^{2}}+\frac{2 n}{2 n+1}(2 x-1) \frac{d u}{d x}+\frac{n(n-1)}{(2 n+1)^{2}} u=0 \ldots \ldots \text { (4) }
$$

which is readily seen to be the differential equation of the ordinary hypergeometric function

$$
F\left(\frac{n-1}{2 n+1}, \frac{n}{2 n+1} ; \frac{2 n}{2 n+1} ; x\right) .
$$

§3. Let us consider in detail the case $n=3$, the algebraic form taken being $\quad s^{2}=1+z^{7}$. 
The hypergeometric equation (4) for this particular case takes the form

$$
49 x(x-1) \frac{d^{2} u}{d x^{2}}+42(2 x-1) \frac{d u}{d x}+6 u=0
$$

If we put $a=1 / 7$ (since multiples of $1 / 7$ will appear often in the following calculations), four solutions of equations (5) will be

$$
\begin{aligned}
& P=F(2 a, 3 a ; 6 a ; x) \\
& Q=x^{a} F(3 a, 4 a ; 8 a ; x)
\end{aligned}
$$

which are valid at $x=0$, and

$$
\begin{aligned}
& R=x^{-2 a} F\left(2 a, 3 a ; 6 a ; x^{-1}\right) \\
& S=x^{-3} F\left(3 a, 4 a ; 8 a ; x^{-1}\right)
\end{aligned}
$$

which are valid at $x=\infty$.

These solutions are connected by the relations :

$$
\begin{aligned}
& P=\frac{\Gamma(6 a) \cdot \Gamma(a)}{\Gamma(3 a) \cdot \Gamma(4 a)}(\exp \pm 2 \pi a i) R+\frac{\Gamma(6 a) \cdot \Gamma(-a)}{\Gamma(2 a) \cdot \Gamma(3 a)}(\exp \pm 3 \pi a i) S \\
& Q=\frac{\Gamma(a) \cdot \Gamma(8 a)}{\Gamma(5 a) \cdot \Gamma(4 a)}(\exp \pm 3 \pi a i) R+\frac{\Gamma(-a) \cdot \Gamma(8 a)}{\Gamma(4 a) \cdot \Gamma(3 a)}(\exp \pm 4 \pi a i) S
\end{aligned}
$$

where in either case the upper or lower sign is taken according as the imaginary part of $x$ is positive or negative.

Take $t=Q / P$ for the uniformising variable and let $t$ denote $Q / P$ at $i \infty$ and $t^{\prime}$ denote $Q / P$ at $-i \infty$; then

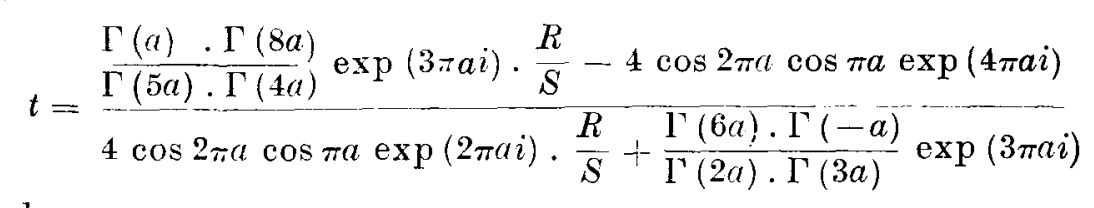

and

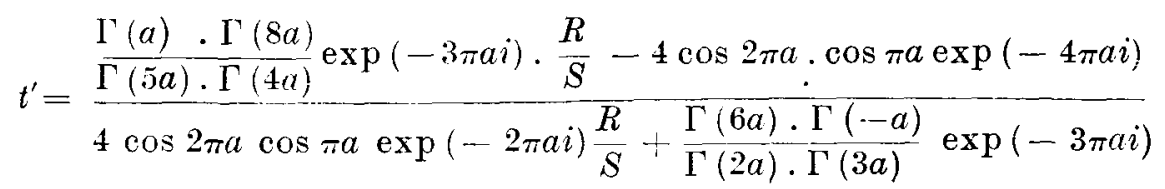

If we eliminate $R / S$ from these equations we get

$$
t^{\prime}=\frac{[4 \cos \pi a \cos 2 \pi a \exp (-2 \pi a i)-2 \cos \pi a] t+\frac{\Gamma(a) \cdot \Gamma(8 a)}{\Gamma(5 a) \cdot \Gamma 4 a} 2 i \sin \pi a}{\frac{\Gamma(6 a) \cdot \Gamma(-a)}{\Gamma(2 a) \cdot \Gamma(3 a)} 2 i \sin \pi a \cdot t+[4 \cos \pi a \cos 2 \pi a \exp (2 \pi a i)-2 \cos \pi a]}
$$


or

$$
t_{1}^{\prime}=\frac{2 \cos \pi a \exp (-4 \pi a i) \cdot t_{1}-8 \cos ^{2} \pi a \cos 2 \pi a \cdot 2 i \sin \pi a}{2 i \sin \pi a t_{1}+\frac{2 \cos \pi a \exp 4 \pi a i}{2}}
$$

where

$$
t_{1}=\frac{\Gamma(6 a) \cdot \Gamma(-a)}{\Gamma(2 a) \cdot \Gamma(3 a)} t
$$

This is the transformation which the quotient of two solutions of equation (5) undergoes when $x$ passes from $i \infty$ to $-i \infty$ making a circuit round the singularity $x=1$.

When $x$ makes a circuit round the origin in the $x$-plane, i.e. $s$ makes a circuit round the singularity -1 in the $s$-plane, then the quotient of two solutions of the equation undergoes the transformation

$$
t_{1}^{\prime}=\exp (2 \pi a i) \cdot t_{1} .
$$

Before proceeding any further let us put equation (6) in a more convenient form by taking

$$
t_{1}=i \cot \pi a \exp (3 \pi a i) t_{2}
$$

we then have the two transformations

and

$$
t_{2}^{\prime}=\frac{\exp (-\pi a i) \cdot t_{2}-8 \sin ^{2} \pi a \cos 2 \pi a}{\exp (-\pi a i) \cdot t_{2}-1}
$$

$$
t_{2}^{\prime}=\exp (2 \pi a i) \cdot t_{2}
$$

or again, taking

$$
t_{\mathrm{a}}=\left(8 \sin ^{2} \pi a \cdot \cos 2 \pi a\right)^{\frac{1}{2}} t_{3},
$$

we get the transformations

$$
t_{3}^{\prime}=\frac{\exp (-\pi a i)\left(8 \sin ^{2} \pi a \cos 2 \pi a\right)^{-\frac{1}{2}} t_{3}-1}{\exp (-\pi a i) t_{3}-\left(8 \sin ^{2} \pi a \cos 2 \pi a\right)^{-\frac{1}{2}}}
$$

and $\quad t^{\prime}{ }_{3}=\exp (2 \pi a i) \cdot t_{3}$.

If we call these transformations $L$ and $M$ respectively, it can easily be shown that $L M^{-1}$ is the transformation which the quotient of two solutions of (5) undergoes when $z$ makes two successive circuits: (i)

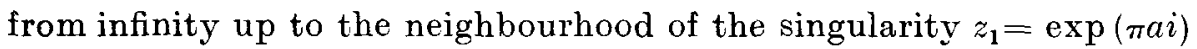
round $z_{1}$, and back to infinity; (ii) from infinity to the neighbourhood of the singularity $z_{2}=\exp (3 \pi a i)$, round it and to infinity again. 
This is better seen in Fig. 1. If we call $E_{1}$ and $E_{2}$ the transformations which the quotient undergoes when describing the two contours in the figure we have $E_{1} E_{2}=L M^{-1}$, so that

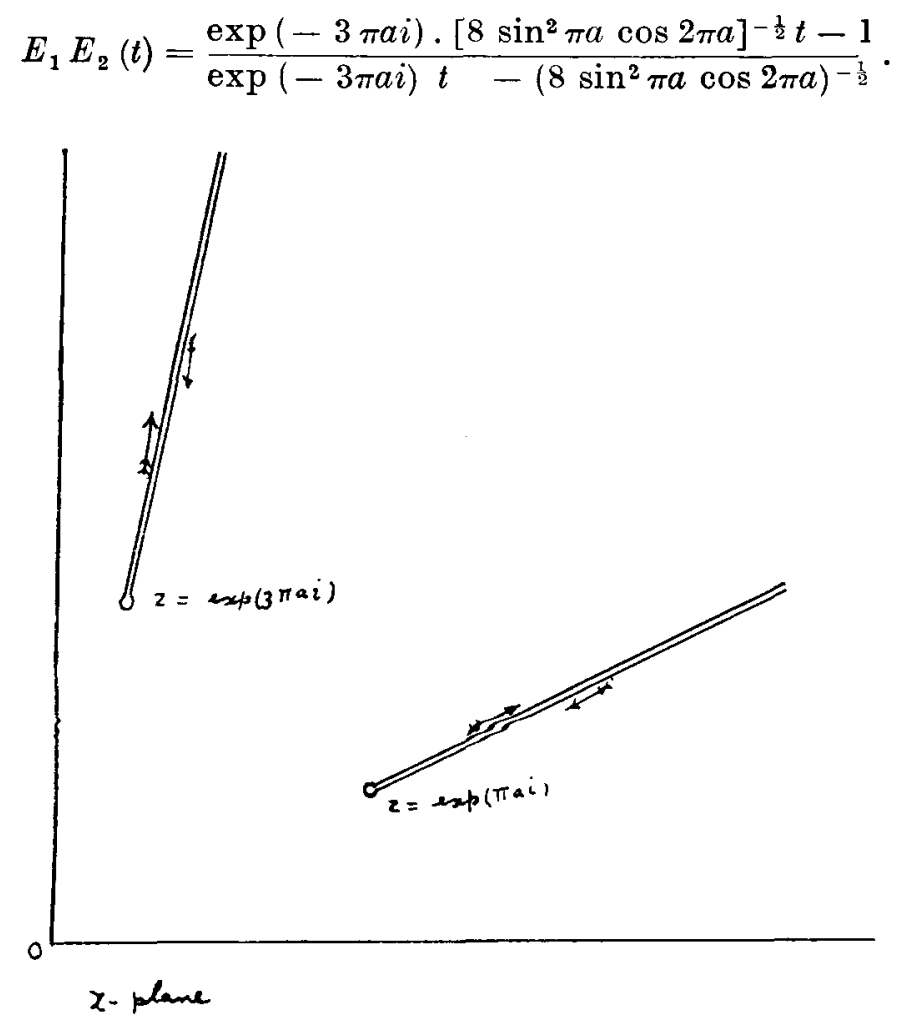

Fig. 1

§4. Consider now the seven transformations given by

$$
S_{r}(t)=\frac{a t-\exp (4 n+1) \pi a i / 2}{\exp \{-(4 n+1) \pi a i / 2\} t-a},
$$

where $n=0,1,2, \ldots 6$ and $r=1,2, \ldots 7$ respectively. The value of $\alpha$ is given by

$$
a=(2 \cos \pi a-1)^{-\frac{1}{2}} \text {. }
$$

It is easily verified that this set of transformations satisfies the following conditions.

(i) They are elliptic transformations of period 2, i.e. they are selfinverse transformations.

(ii) The same is true for the compound transformation $S_{7} S_{6} \ldots S_{1}$. 
(iii) The unit circle is invariant with respect to each of them and hence is invariant under any combination of these transformations.

(iv) The fixed points (double points) of the transformations are

$$
\rho \exp (4 n+1) \pi a i / 2, \quad n=0,1,2, \ldots 6,
$$

and their inverses in the unit circle, where

$$
\rho=(2 \cos \pi a-1)^{-\frac{1}{2}}\left(1-2 \sin \frac{1}{2} \pi a\right) .
$$

Now consider

$$
S_{1} S_{2}(t)=\frac{\left[a^{2}-\exp (-4 \pi a i / 2)\right] t+a(\exp \pi a i / 2-\exp 5 \pi a i / 2)}{\alpha[\exp (-\pi a i / 2)-\exp (-5 \pi a i / 2)] t+\alpha^{2}-\exp 4 \pi a i / 2} .
$$

Comparing this with the expression for $E_{1} E_{2}(t)$ in $\S 3$ we find (after inserting the value of $a$ in (9)) that

$$
E_{1} E_{2} \equiv S_{1} S_{2} \text {. }
$$

Thus we may take as the set of transformations $E_{1}, E_{2} \ldots, E_{7}$ the expressions ${ }^{1}$

$$
E_{r}(t)=\frac{a i-\exp \{(4 n+1) \pi a i / 2\}}{\exp \{-(4 n+1) \pi a i / 2\} t-\alpha}, \quad n=0,1,2, \ldots, 6
$$

where $a=(2 \cos \pi a-1)^{-\frac{1}{2}}$.

§5. In this section the properties of the "fundamental region" of the group generated by the transformations $E_{1} E_{2}, \ldots, E_{1} E_{7}$ will be enumerated briefly.

The fundamental region for the group generated by $E_{1}, E_{2}, \ldots, E_{7}$ is bounded by arcs of circles passing by the double points and cutting the fixed circle orthogonally. This is the heptagon $A B C D E F G$ of figure (2). The genus of the group, i.e. the genus of its fundamental region, is zero, but we shail see that it has sub-groups of genus greater than zero. Now call this heptagon $R_{0}$ and transform it by any of the transformations $E_{1}$ say; then we get another region $R_{1}$ abutting on $R_{0}$ along the side $A B$. The combined region $A C_{1} D_{1} E_{1} F_{1} G_{1} B$ $C D E F G A$ will be the fundamental region for the group of transformations generated by $E_{1} E_{2}, E_{1} E_{3}, \ldots, E_{1} E_{7}$. It has six congruent pairs of sides of the 1st kind, namely, $A C_{1}$ and $B C, C_{1} D_{1}$ and $C D, \ldots$ and, finally, $G_{1} B$ and $G A$, which are congruent by the

${ }^{3}$ This follows from the uniqueness of the group of the differential equation and also from the symmetry of the positions of the contour in the :-plane. 
substitutions $E_{2} E_{1}, E_{3} E_{1}, \ldots E_{7} E_{1}$. All the various vertices congruent and form a cycle the sum of whose angles is $2 \pi$. The genus of this fundamental region is three.

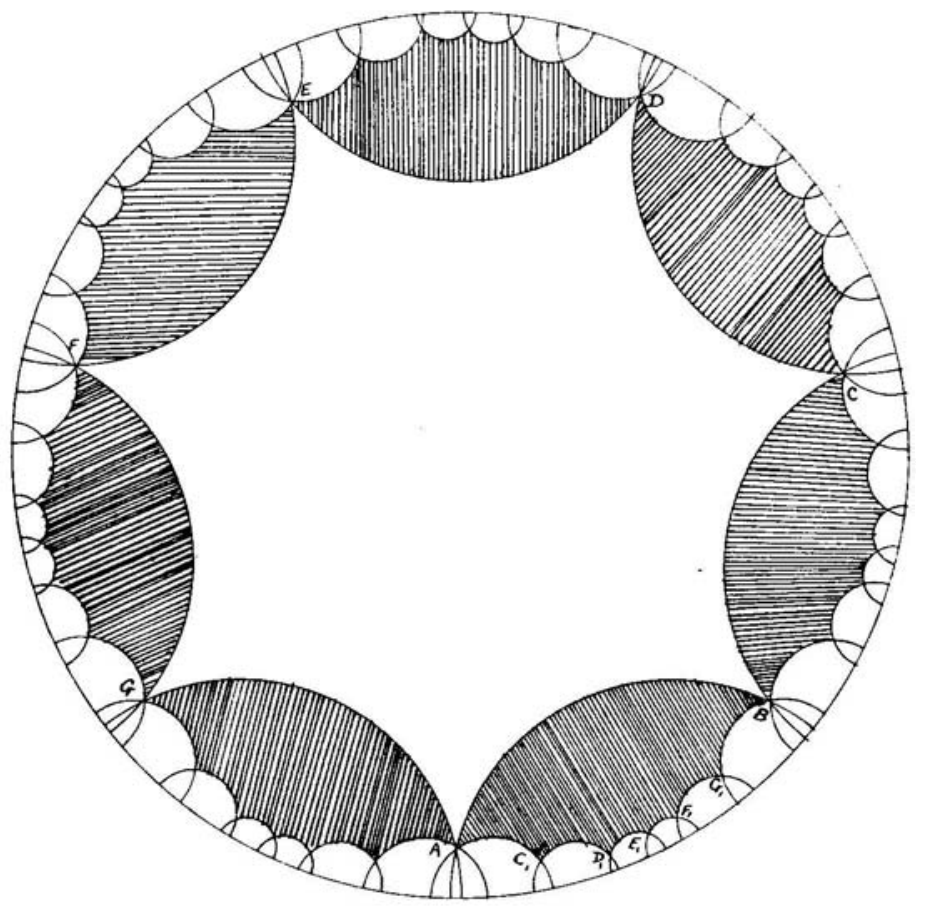

Fig. 2

Now the interior of the region $A B C D E F G$ is the conformal representation in the $t$ plane of the whole $z$-plane bounded by cuts drawn radially from the finite singularities to infinity (which is another singular point in our case), the sides of the heptagon being the transforms of these cuts. Now adjoin to this heptagon the neighbouring one $R_{1}$, and the resulting curvilinear figure will be the conformal representation on the $t$-plane of two $z$-planes joined together along one of the branch lines $\left(e_{r}, \infty\right)$. In fact it is the dissected Riemann surface of the curve

$$
s^{2}=z^{7}+1 \text {. }
$$

The uniformisation of the functions connected with the form $s^{2}=1+z^{7}$ is effected by automorphic functions of the group, the construction of these functions being a matter of straightforward calculation. 